\title{
Review Healthcare Training or Educational Materials
}

National Cancer Institute

\section{Source}

National Cancer Institute. Review Healthcare Training or Educational Materials. NCI

Thesaurus. Code C128499.

The healthcare provider or patient must review certain training or educational materials. 